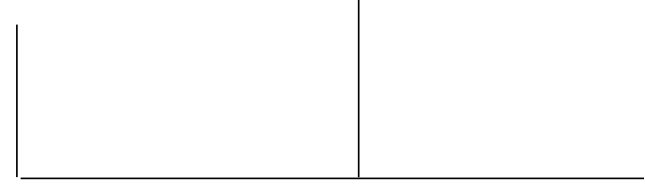

Rev. Latinoam. Psicopat. Fund., VIII, 1, 64-72

\title{
Concepto de padres combinados
}

\author{
José de Jesús Gonzáles Núñez, Joseph Simo, \\ Carmen Gamietea, Carlos Caudillo, Susana Zarco
}

En el presente artículo se presenta el concepto de padres combinados que se refiere a la percepción indiferenciada del bebé acerca del coito parental, en donde el niño percibe que el padre está contenido en la madre, por lo que no reconoce las funciones y atributos del padre, ya que pertenecen a la madre.

Se distingue la ubicación teórica del concepto que corresponde a la fase esquizoparanoide de Melanie Klein, los mecanismos de defensa utilizados, así como las manifestaciones clínicas. Se expone un caso que muestra que la persona que posee la fantasía de padres combinados manifiesta una percepción parcial de los objetos, así como una gran falla en la diferenciación de los mismos, que crea la fantasía en la persona de que él mismo los reúne y los combina, situación que le resulta angustiosa, experimentando envidia y anhelo hacia los padres combinados, así como temor a la retaliación por haber dirigido hacia ellos sus impulsos sádicos.

Palabras claves: Bebés, padres combinados, angústia, Melanie Klein 


\section{Padres combinados}

El concepto de padres combinados fue utilizado inicialmente por Klein (1987) para describir la percepción indiferenciada del bebé acerca del coito parental.

El niño se encuentra en una primitiva etapa de desarrollo, en donde no puede establecer una diferenciación entre las introyecciones de ambos padres, y percibe al pene del padre como parte del cuerpo materno, y más específicamente percibe al padre como componente del self de la madre.

Por lo tanto, la madre crea y construye al padre, y en esa medida, el niño no lo reconoce como objeto diferenciado, sino como parte de la madre. Como consecuencia, el niño no es capaz de percibir ni reconocer las funciones propias del padre, ya que siempre pertenecen a la madre.

Epigenéticamente, el concepto de padres combinados corresponde a la fase esquizoparanoide de Melanie Klein, fase en la cual predominan en el funcionamiento mental del niño, los mecanismos de proyección, escisión e identificación proyectiva.

Por medio del mecanismo de proyección, el niño se coloca afuera del pecho de la madre, en la medida que este se convierte en una amenaza para el yo.

De esta manera, el impulso destructivo es proyectado hacia fuera.

Con la escisión logra separar el pecho bueno del malo, y mientras el malo es proyectado, el bueno es el que contrarresta el proceso de escisión y contribuye a la construcción del yo.

A los impulsos orales sádicos, se agregan los de la fase anal sádica, así como las fantasías uretrales. Antes de despertar los instintos genitales, el niño comienza a desarrollar teorías acerca de la copulación, que postulan que durante el coito, la madre incorpora al pene de padre - y al padre en su totalidad. Con el predominio de los impulsos sádicos, el niño desea penetrar en el cuerpo materno y destruir el pene y al propio padre que ahí se encuentra contenido, ya que los padres disfrutan de un coito interminable, de una unión continua de la que él no se siente parte.

En la fantasía de los padres combinados, no existe en el niño una noción de objeto total, por lo que los padres se perciben como objetos parciales que se unen en contra del sujeto.

Así mismo, el infante desea penetrar el cuerpo de la madre para dañar los órganos y objetos que ahí encuentre, motivado por la envidia, ira, frustración y 
por sus deseos de apropiarse de ellos. Esta depredación es la fantasía terrorífica sobre el cuerpo materno que contiene al pene del padre. Esta fantasía le genera al niño un terror profundo que se incrementa al sentir que la madre y los objetos que contiene se vengarán de él. A su vez no puede renunciar a ellos porque los necesita para llenar el vacío que dejó al proyectar en el cuerpo de la madre, todas esas partes indeseadas de sí mismo, fusionadas con las partes deseadas de sí mismo. Requiere reintroyectar partes buenas del objeto pero éstas a su vez se encuentran fusionadas con partes malas, a las que dañó. Para no quedar vacío tiene que introyectar objetos malos que se convierten en perseguidores internos, perseguidores que están dentro de sí mismo, con una idea original de controlarlos, pero al sentirse controlado por ellos experimenta terribles momentos de paranoia.

El objetivo del infante es acabar con los padres combinados en una fantasía sádica omnipotente. Sin embargo los dota de instrumentos de destrucción mutua, transformándolos en objetos poseedores de características bestiales para atormentarse y destruirse entre ellos en la copulación. Así, cada coito es experimentado por el niño como un acto sumamente peligroso en el que quienes están "combatiendo" se unirán para aniquilarlo.

La fantasía de los padres combinados implica el reconocer a la madre como un objeto parcial, pero en vías de convertirse en total, donde el niño percibe que lo bueno y lo malo proviene del mismo objeto - madre; sin embargo no logra una clara diferenciación con la figura de objeto - padre. En su fantasía el pene o el padre son parte de la madre, y la idealización que hace de ella lo lleva a concebirla como la que tiene todo lo deseado: pecho, pene, bebés (Segal, 1964). Los ataques de envidia y las proyecciones personales pueden convertir a la madre en una perseguidora terrorífica.

Conforme el niño avanza a la siguiente etapa llamada por Klein, posición depresiva, lo bueno y deseado que le atribuye a los padres combinados, predominará sobre lo terrorífico y perseguidor, lo cual le permitirá percibir a la madre y al padre como objetos separados, objetos totales. Por lo tanto, la angustia persecutoria disminuye, al igual que el miedo a la aniquilación.

Al surgir posteriormente ansiedades edípicas, la fantasía de padres combinados puede reactivarse como negación del coito parental, causando ansiedades persecutorias que llegan a producir neosexualidades (McDougall, 1982).

\section{Creacion del objeto y padres combinados}

Según Simo (1991) el proceso de padres combinados se ubica en la quinta fase de desarrollo de la creación del objeto. El postula el siguiente esquema: 
1) El útero materno, cuando el bebé está envuelto por el objeto absoluto.

2) La explosión del objeto absoluto, representada por el trauma del nacimiento

3) El niño escinde el falo, siguiendo las experiencias dadas por el principio de realidad. Existe un proceso de escisión y fragmentación continuos del objeto y del sujeto.

4) Al proceso de fragmentación del objeto, le sigue un proceso de "fertilización orgánica" de los fragmentos, que se enriquecen con información del mundo interno y externo y transforman el contenido y la manera de percibir del bebé.

5) El niño une los pedazos del sujeto-objeto en una gestalt total que va a producir el objeto. Es la fase frankisteiniana, en la cual el niño une esos pedazos y crea un objeto total que percibe: a) Como una unidad corporal (la forma final del objeto), b) como un objeto total existente, y c) como contrapartida del yo. Este objeto va a evolucionar, experimentando una serie de transformaciones producidas por la maduración del aparato psíquico y la capacidad de metabolizar e integrar la información que proviene del mundo interno, del mundo externo y de la introyección de las proyecciones de los objetos que lo rodean.

Desde el punto de vista evolutivo, en esta fase es en la cual se integra al objeto, aun no como totalmente diferenciado, por lo que adquiere las caracteristicas combinadas de ambos objetos parentales.

El niño al encontrarse por primera vez con las evidencias anatómicas de los sexos, experimenta un rechazo hacia dichas evidencias, y las reemplaza por la fantasía de padres combinados, que es una elaboración infantil de la escena primaria, percibida sádicamente, en las cual la madre incorpora en su cuerpo el pene del padre (Simo, 1993).

\section{Padres combinados e identificacion proyectiva}

La identificación proyectiva es una forma de defensa ante la angustia que experimenta el niño, al poseer partes malas que necesita expulsar sobre el objeto y al reconocer partes buenas del objeto, que necesita asimilar como propias.

Klein (1946) propone como término de identificación proyectiva, al mecanismo por medio del cual el sujeto se relaciona agresivamente con su objeto, expulsa de sí las partes malas y las coloca en el objeto, y toma las partes buenas del objeto para sí mismo. La finalidad es de atacar, apoderarse y controlar, desde su interior al objeto.

Es una fantasía alejada de la conciencia que supone la creencia de que ciertos aspectos del self están localizados en otra parte, con el consiguiente 
vaciamiento y debilitamiento del sentimiento de sí y de la identidad, que tiene alcances de despersonalización; pueden sobrevenir profundos sentimientos de extravío, o una sensación de aprisionamiento (Hinshelwood, 1992).

La fantasía angustiosa es de hallarse aprisionado y perseguido en el interior del cuerpo materno, o también la identificación proyectiva puede provocar que la introyección sea sentida como una penetración forzada desde el exterior al interior como castigo por una proyección violenta. (Laplanche y Pontalis, 1968). Otro peligro es que el yo se encuentre debilitado y empobrecido en la medida en que puede perder, en la identificación proyectiva partes buenas de sí mismo. Es aquí donde la fortaleza del rol paterno puede ser rescatadora y ayudar a la diferenciación del yo y los objetos contenidos en el self.

Bion $(1959,1962)$ menciona que hay identificación proyectiva normal y anormal, dependiendo del grado de violencia con la que se realiza dicho mecanismo. La identificación proyectiva anormal sería por:

a) El grado de odio y violencia contenidos en la escisión

b) El control omnipotente y fusión con el objeto

c) El monto de lo perdido del yo

d) La meta específica de destruir en especial la realidad interna

La identificación proyectiva normal persigue la meta de obtener comunicación y empatía con el objeto, desempeña su papel en la participación de la realidad social. Es muy parecida a las funciones yoicas de relaciones objetales y prueba de realidad. Busca evacuar un estado psíquico penoso, lo que conduce mediante la fantasía, la entrada forzada en un objeto, con el afán de obtener alivio inmediato por medio del objeto, además, introducir en el objeto un estado psíquico como medio para comunicarse con él. El estado penoso con el objeto y la comunicación se conectan con diferentes defensas y producen diferentes efectos en la fantasía, sobre el objeto y el yo. Se convierten en patología por la gran violencia y omnipotencia con la que se lleva a cabo, tanto en frecuencia como en intensidad (Hinshelwood, 1992).

Rosenfeld (1983), citado por Hinshelwood (1992) refiere que los propósitos de la identificación proyectiva son:

1) Propósito defensivo (para liberar al self de partes indeseadas)

a) Intrusión omnipotente resultando fusión o confusión con el objeto

b) Fantasía de vivir pasivamente dentro del objeto (parasitismo)

c) Creencia de una identidad de sentimiento con el objeto (simbiosis)

d) Expulsión de tensión por parte de alguien que ha sido traumatizado cuando niño por intrusiones violentas

2) Propósito de comunicación

a) Vía de acceso hasta un objeto al que se cree distante 
b) Inversión del vínculo hijo-progenitor

c) Identificación con similitudes encontradas en el objeto con fines narcisistas

3) Propósito de reconocimiento de objetos para identificarse con ellos (empatía) La identificación proyectiva interviene en la construcción de la identidad del self y de los objetos. Su utilización puede traer consecuencias para el sujeto, como la disociación del self experimentado de varias formas:

a) Sensación de estar despedazado

b) sensación de futilidad

d) no sentirse persona (despersonalización)

e) sensación de que le han arrancado a la fuerza o estar aprisionado y ser controlado (claustrofobia)

f) aferramiento al objeto donde se ha localizado partes del self

g) angustia por haber dañado al objeto en la intrusión y control

h) angustia por la retaliación del objeto

i) el destino del objeto en la identificación proyectiva patológica es el destino del self perdido, que se puede percibir como ajeno y perseguidor.

Estas ideas llevan a pensar en que la desconexión de pensamientos en la mentes es un ataque activo a los vínculos entre contenidos mentales.

Bion (1959) lo comparó con la vinculación edípica, en donde el ataque al vínculo edípico entre contenidos mentales contradictorios, es un ataque a la pareja parental vivida como objetos-partes. Se trata de la vinculación de la boca con el pecho, o de la vagina con el pene.

Estas fantasías son experimentadas corporalmente por medio de la succión y del sexo. Con la idea de colocar un elemento dentro del otro, en la relación continente - contenido hay un acoplamiento de los dos objetos.

\section{Manifestaciones clinicas de los padres combinados}

El paciente $\mathrm{R}$ tiene 43 años y durante su proceso psicoterapéutico ha manifestado un gran temor a la sexualidad, actualmente no se acerca a su pareja, sino en muy contadas ocasiones, no más de 3 o 4 encuentros sexuales en un año. Una de las fantasías más profundas que ha revelado es el temor a destruir de tal forma los genitales de su pareja, que a la vez destruya los suyos, le da miedo que cuando esté en el coito, se quede en una relación muy prolongada y no se pueda separar, lo que le provoca angustia y sobre todo sensaciones de terror. La relación sexual se ha cargado de tal cantidad de envidia, que considera que el coito puede conducirle a apoderarse de los pechos maternos, proyectados en la pareja y así 
destruir a la madre internalizada y a la pareja. Como consecuencia, el amor de R solo tiene lugar en la abstinencia sexual.

El paciente frecuentemente representa una escena mental en donde los padres están unidos y a la vez escindidos en partes, con la alucinación angustiosa de no saber quién es quién y que partes representa a cada uno. Esta angustia y esta confusión se confirman en un sueño en el cual relata que se vio en una relación sexual con alguien desconocido, él veía los genitales de ella y de pronto, misteriosamente, empezaba a aparecer un pene. En ese momento se angustió:

1) Al no saber si él estaba viendo unos genitales femeninos y cómo era que se transformaban en un pene amenazador y

2) la angustia que sentía de ser homosexual o la predicción de que esto le pasaría.

Este sueño nos refleja cómo R posee internalizada la fantasía de padres combinados, con genitales no diferenciados y la percepción parcial de objetos simbólicos amenazantes a su yo. Sin embargo refleja también el deseo de poseer características femeninas, genitales femeninos, vistos en el sueño en la identificación proyectiva y su transformación en genitales masculinos, que penetran a los femeninos en una misma escena.

Otro aspecto importante en la fantasía de padres combinados es el proceso en el que se dio un significado a los "padres" en donde el significado de padre corresponde a una vivencia temprana (previa a la estructuración de la palabra y previa a la solución del conflicto de Edipo). El padre ha sido presentado por la madre como femenino, y en esta manera no reconoce ni permite en el hijo el reconocimiento de las funciones paternas. Por otra parte, la madre juega un papel masculino, que simplemente gratifica las necesidades y funciona con apoyo "sosten".

En el caso de R, la sexualidad fue vivida con angustia y frustración, no logra durante el sueño, la pantalla cálida y segura maternizante del orgasmo, ya que según él, el orgasmo también está contenido en una pantalla igualmente maternizante y cálida que le da protección y seguridad, en ese momento eterno donde la regresión y el descontrol impulsivo de la descarga lo debilitan, y el individuo puede ser destrozado por alguno de los padres (González Núñez, 1997). Aparece la eyaculación precoz como una forma de no tener relaciones sexuales con su pareja y para no sentir la angustia perversa de la no diferenciación.

La fantasía de padres combinados necesariamente implica el asumir a los objetos de forma parcial. Son pecho-pene, leche-semen, etc. Se expande así el control fantaseoso del yo del sujeto que considera que él es quien los reúne y los combina. Aparece también la angustia por la sensación de omnipotencia y la impotencia por no poder manejar la situación.

En el caso de R, veía a su esposa como madre-padre, esposa-hijo, hija-hijo; siempre una combinación indiferenciada, odiada y anhelada. Desea el acto sexual 
para despojar a la madre y al padre de las cualidades que él envidia, pero teme poseerlas, porque a la vez va a ser robado y aniquilado.

Así podemos concluir que en el psiquismo temprano, el infante humano atraviesa por procesos que se manifiestan clínicamente a través de la fantasía angustiosa e indiferenciada en la que se anhela y se destruye a los objetos parciales existentes en ella, con el consecuente temor a la retaliación por haber deseado la destrucción y la posesión de tales objetos.

\section{Referencias}

Bion, W. Ataques al vínculo. In: Volviendo a pensar. Buenos Aires: Hormé, 1959.

Una teoría del pensamiento. In: Volviendo a pensar. Buenos Aires: Hormé, 1962.

GonZÁlez NúÑez, J. J. Sexualidad masculina: normalidad y patología. Conferencia Magistral presentada en el IX Coloquio Nacional: Expresiones de la Sexualidad Masculina: Normalidad y Patología. Museo Nacional de Antropología. México, 1997.

Hinshelwood, R.D. Diccionario del pensamiento kleiniano. Buenos Aires: Amorrortu, 1992.

KLeIN, M. Primeros estadíos del conflicto de Edipo y de la formación del Superyó. In: El psicoanálisis de niños. Obras completas. Madrid: Paidós, 1928. t. II.

Notas sobre algunos mecanismos esquizoides. In: Envidia y gratitud. Obras completas. Madrid: Paidós, 1946. t. III.

Laplanche, J. e Pontalis, J.-B. Diccionario de psicoanálisis. Madrid: Labor, 1968.

McDougall, J. Teatros de la mente. Madrid: Ed. Julián Yébenez. S.A., 1982.

Segal, H. Introducción a la obra de Melanie Klein. Madrid: Paidós, 1964.

Simo, J. El ocaso de la omnipotencia: la creación del objeto. In: Simbiosis, individuación y creación del objeto. México: Editorial Instituto de Investigación en Psicología Clínica y Social, 1991.

El hombre máquina en el laberinto del deseo. In: Alteraciones afectivas en la psicopatología sexual masculina. México: Editorial Instituto de Investigación en Psicología Clínica y Social, 1993.

\section{Resumens}

No presente trabalho apresenta-se o conceito de pais combinados que se refere à percepção indiferenciada do bebê a respeito do coito parental, no qual o bebê 
percebe o pai como contido na mãe e, portanto, não reconhece as funções e atributos do pai já que pertencem à mãe.

Distingue-se que a orientação teórica do conceito forma parte da fase esquizoparanóide de Melanie Klein, assim como os mecanismos de defesa utilizados e as manifestações clínicas. Relata-se um caso no qual a pessoa possuidora da fantasia de pais combinados manifesta uma percepção parcial dos objetos, falha na diferenciação dos mesmos e cria a fantasia de que ele mesmo os reúne e os combina, situação essa que resulta angustiosa, experimentando inveja, anseio para os pais combinados e temor à retaliação por haver dirigido impulsos sádicos para com eles.

Palavras-chave: Bebês, pais combinados, angústia, Melanie Klein

Cet article présente le concept de parents combinés, qui se réfère à la perception indifférenciée qu'a le bébé du coüt parental. L'enfant perçoit que le père est contenu dans la mère, et ne reconnaît pas les fonctions et attributs paternels puisqu'ils appartiennent à la mère.

L'article expose les bases théoriques du concept correspondant au stade schizoparanoïde de Melanie Klein, les mécanismes de défense employés et les manifestations cliniques. Il est présenté un cas qui montre que celui qui a le fantasme des parents combinés manifeste une perception partielle des objets et une grande faille dans la différenciation de ces derniers, créant un fantasme où il est le seul qui peut les rassembler et les unir. Cette situation provoque de l'angoisse, de l'envie et de l'espoir vis-à-vis des parents combinés, tout autant qu'une crainte de représailles pour avoir dirigé vers eux des pulsions sadiques.

Mots-clés: Bébés, parents combinés, angoisse, Melanie Klein

This article presents the concept of combined parents, which explains a baby's undifferentiated perception of its parent's sexual intercourse. The baby perceives that the mother contains the father and, for this reason, does not recognize the functions and attributes of the father, since the attributes belong to the mother.

The article distinguishes the theoretical aspect of the concept, which corresponds to Melanie Klein's esquizo-paranoide position, the defense mechanisms used, and the clinical manifestations involved. The article also discusses a case that shows how a person with the fantasy of combined parents manifests a partial perception of objects, and thus has serious difficulties in understanding the difference between his parents, creating the fantasy that this person himself unites and combines them. This situation brings about distress, envy and hopes concerning the combined parents, as well as a fear of the parents' retaliation for the child's having directed his sadistic impulses toward them.

Key words: Babies, combined parents, anxiety, Melanie Klein

Versão inicial recebida em janeiro de 2003

Versão revisada recebida em setembro de 2003 